\title{
Habermas, identidad moderna y mejora humana. $¿$ El camino a seguir?
}

\author{
Habermas, modernidentity and buman enbancement. \\ Is the way forward?
}

FRANCISCO JAVIER LÓPEZ-FRÍAS

UNIVERSIDAD DE VALENCIA

Artículo recibido: 25 marzo 2013

Solicitud de revisión: 15 julio 2013

Artículo aceptado: 16 julio 2013

\section{Resumen}

La finalidad de este artículo es triple. Primero, las secciones 1 y 2 expondrán la estructura del argumento de Jürgen Habermas respecto a las tecnologías de mejora humana. Para ello, éste será descompuesto en tres partes, que son sus pilares básicos: a) los sujetos diseñados no pueden auto-concebirse a sí mismos como autores únicos de sus vidas; b) la distinción kantiana entre objetos y personas se desvanece como consecuencia del modo en que los sujetos modificados experiencian su cuerpo;c) la relación entre los padres y su descendencia se vuelve asimétrica, de modo que es contraria a las normas de la acción comunicativa. Segundo, en la sección 3, que es concebida como un diálogo entre Habermas y sus críticos, se presentarán algunas objeciones teóricas a su argumento. Tercero, la sección 4 analizará la validez de los argumentos habermasianos y afirmará firmemente que la posición de Habermas arroja luz sobre el modo correcto de analizar las tecnologías de mejora humana desde un punto de vista moral. A saber, en función de la influencia que tienen sobre el modo en que los sujetos se auto-conciben como seres autónomos y responsables.

Palabras clave: Mejora humana, Jürgen Habermas, autonomía, modernidad.

\begin{abstract}
The aim of this paper is threefold. First, the structure of Jürgen Habermas' argument on human enhancement technologies shall be exposed in sections 1 and 2 . In order to do so, this paper shall discompose Habermas' argument in the following three parts, which will be taken to be its central pillars: a) designed people cannot self-conceive themselves as the sole authors of their lives; b) the Kantian distinction between objects and persons falls apart due to the way in which modified people experience their bodies; c) the relationship between parents and their modified offspring turns into an asymmetric one, so it is contrary to the rules of communicative action. Secondly, several objections to Habermas' claims shall be exposed in section 3 , which is mainly conceived as a dialogue between Habermas and his critics. Thirdly, section 4 shall analyse the validity ofHabermas' claims. It
\end{abstract}


shall firmly argue that his position sheds light on the right way to analyse human enhancement technologies from a moral point of view.

Key Words: Human enhancement, autonomy, Jürgen Habermas, modernity.

\section{MODERNIDAD Y MEJORA HUMANA. HUMANIZACIÓN DESNATURALIZADORA}

\subsection{Las promesas de la modernidad}

Mirando al pasado de la filosofía occidental damos muy fácilmente con las raíces modernas del debate ético en torno a la mejora humana. Puede decirse que las filosofías de René Descartes y Francis Bacon, como padres de la Modernidad y defensores de la ciencia, ya contenían la esperanza de alterar técnicamente la naturaleza humana. Un anhelo que comenzó a hacerse realidad, aproximadamente, en los años sesenta tras la decodificación del genoma humano y los posteriores avances en técnicas biomédicas que siguieron a dicho descubrimiento. Desde entonces, el debate ético-filosófico en torno a los avances de la genética, la biomedicina, las neurociencias... no ha parado, pues éstos parecen socavar -o al menos estar estrechamente relacionados con- los fundamentos que parecen esenciales para nuestra identificación y constitución como sujetos modernos.

Atendiendo al discurso transhumanista actual, la mejora de nuestra naturaleza como algo propiamente moderno no sólo es una antigua promesa alcanzada por la ciencia, sino una cuestión ligada a lo más propio del sujeto moderno: la autonomía y la capacidad que cada uno debe poseer para forjar a su gusto su propia identidad (Elliot, 2003). Por ello, la modificación de la propia naturaleza es concebida como un cuasi-derecho individual, una opción que cada cual debe tener la posibilidad de realizar. Así, desde una posición transhumanista, no debe ponerse freno a la aplicación de la ciencia en la sociedad -además, es consustancial a la modernidad que los prejuicios y miedos no frenen el avance de la razón a través del conocimiento científico. ${ }^{1}$

1 En referencia clara al mundo moderno, una idea ampliamente extendida afirma que toda innovación tecnológica debe «ser manejada» por esa mano invisible que dirige el mercado. De esta manera también las técnicas genéticas de modificación de la naturaleza humana, tal y como afirma Robert Nozick, deben quedar al designio de la ley de la oferta y la demanda de un «mercado genético», que parece ser un juez mucho más equitativo que aquellos estados de principios del siglo xx que trataron de poner en práctica políticas eugenésicas. 


\subsection{Los monstruos de la razón}

Al igual que las posibilidades modificadoras de la naturaleza humana de la ciencia moderna fueron predichas con mucha anterioridad, también los miedos y preguntas que éstas despertaban fueron elaborados antes de que la Escuela de Frankfurt formulara su conocida teoría de la dialéctica de la Ilustración -y también antes de que la biomedicina tuviera algún tipo de entidad. Concretamente, durante el Romanticismo, en el que los miedos hacia el afán de dominio de la ciencia eran una parte central de las críticas al proyecto utópico racional de la modernidad (Turney 1998: 37).

Puede decirse que el mito que verdaderamente caló en las conciencias de las gente y que, de hecho, aún está muy presente en la actualidad, fue, sin duda, aquel Frankenstein creado por Mary Shelley (Cortina, 2011: 3537). Sin embargo, otra obra de ficción aporta, a día de hoy, las bases emotivas que nos llevan a reaccionar con ciertos reparos ante la posibilidad de mejorar la naturaleza humana, a través la intervención técnica de la ciencia: Un mundo feliz de Aldous Huxley. Bajo la influencia de la imagen del mundo predicha por el novelista inglés, muchos han concebido el debate en torno a la mejora como una cuestión que gira única y exclusivamente alrededor del problema de la autonomía.

Entre ellos se encuentra Jürgen Habermas y su obra El futuro de la naturaleza bumana, que es un libro esencial para el debate bioético en torno a la mejora (Habermas, 2002: 95). Si bien el texto de Habermas ha recibido multitud de críticas, muy duras algunas de ellas, ello no quita que, primero, su argumento merezca analizarse con mucha más precisión de lo que se hace -principalmente desde el ámbito anglo-americano. Segundo, que sea un libro cargado de intuiciones geniales, a pesar de, esto es importante de remarcar, haber sido escrito en una época bastante temprana del debate. Tercero, el impresionante esfuerzo que supone para Habermas tratar de aplicar su ética del discurso esencialmente deontológica a solucionar un problema tan concreto -y con tantos recuerdos a las prácticas eugenésicas nazis que su país sufrió.

Este texto analizará el debate de la mejora humana como fenómeno moderno, siguiendo la discusión que Habermas comenzó y que nos lleva por unos derroteros algo distintos de los que ha trazado la bioética angloamericana -aunque, por suerte para la filosofía, los caminos de ambas han acabado cruzándose en autores de una formación «mixta» como Allen Buchanan o Nicholas Agar. 


\section{EL ARGUMENTO DE HABERMAS: EL FUTURO \\ DE LA NATURALEZA HUMANA. UN TEXTO BÁSICO

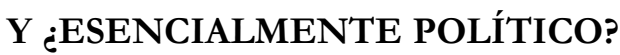

A juicio de Eduardo Mendieta (2002:91), el texto que Jürgen Habermas nos presenta en El futuro de la naturaleza bumana no pertenece ni al terreno de la filosofía ni al de la teoría social, sino que se trata, principal y esencialmente, de una obra política. De hecho, afirma este intérprete del filósofo alemán, el libro debería tener «escritos políticos» como subtítulo. Quiero utilizar esta propuesta interpretativa como comienzo de este apartado, ya que trataré de mostrar que es falsa, pues El futuro de la naturaleza bumana es, principalmente, una obra moral y ética -siguiendo la división tripartita de la razón práctica que Habermas ha ido esbozando a lo largo de su obra.

\subsection{Liberalismo político y mejora humana}

Según Eduardo Mendieta, Habermas ofrece un argumento filosófico, simplemente, a modo de apoyo para lograr unas metas políticas. Es decir, la filosofía es utilizada como un medio para mostrar cómo deberíamos arreglar el problema de la mejora desde un punto de vista jurídico-administrativo. Para él, esta es la posición más acertada respecto a las técnicas de mejora humana, pues la tarea de analizarlas moralmente le parece un lujo que no podemos permitirnos; son «una irrelevancia que nos distrae» (Mendieta, 2002: 111). Lo necesario y adecuado es realizar un análisis en clave jurídico-política, que aporte las bases normativas necesarias para que los individuos no vean sus libertades socavadas en el desarrollo de esa gran «revolución de la información» de la que, como afirma Jeremy Rifkin (1999), el biotecnológico es sólo una parte más.

Así, de acuerdo con la propuesta de Mendieta, el núcleo del argumento habermasiano radica en la supervivencia de las condiciones que nos permiten concebirnos como sujetos políticos típicamente modernos, es decir, como seres responsables y autónomos. Por ello, afirma: «el rechazo de la eugenesia por medios jurídicos -que persigue Habermas- se convierte en un "acto político" que busca conservar la estructura de la modernidad política» (Mendieta, 2002: 107). De hecho, el propio filósofo alemán asegura que su texto es «un acto político de acción moral autorreferente para garantizar la existencia de unas condiciones de conservación de la autocomprensión práctica de la modernidad» (Habermas, 2002: 41). 
Si bien la conservación de las condiciones de la modernidad política es el motivo fundamental del pensamiento de Habermas respecto a la irrupción de las biotecnologías, principalmente, porque son los principios filosóficos que sustentan la modernidad política -dialógicamente fundamentados a lo largo de su carrera filosófica- aquellos que le permiten a Habermas elaborar un argumento posmetafísico (Habermas, 2002: 111) que se libre de tener que abrazar una concepción metafísica del mundo determinada, no obstante, dichas condiciones son de naturaleza filosófica.

Para mostrar la naturaleza moral del texto habermasiano expondré su complejo argumento tratando de mostrar todas sus aristas, pues suele ser habitual, sobre todo, en el ámbito anglo-americano, que éste se reduzca a un par de líneas y se simplifique para hacerlo fácil de rebatir ${ }^{2}$. Sin embargo, esto muestra que, primero, muchos autores no lo comprenden adecuadamente por una simple cuestión de desconocimiento de la tradición filosófica occidental. Segundo, no se percibe la importancia de hacer ese análisis moral que el filósofo alemán, entre otros muchos autores que habitualmente son denominados «bioconservadores», reclama con el fin de encontrar una guía normativa desde la que afrontar el desafío que las biotecnologías nos presentan. Como afirma Jonathan Pugh de la Universidad de Oxford, Habermas «merece un juicio justo».

\subsection{El argumento habermasiano: de la autonomía a una cuestión} de identidad en tres pasos

Sobre el argumento habermasiano cabe destacar que, en principio, se formula en contra de las intervenciones genéticas de mejora pre-natales, que son aquellas que se discuten vivamente en el momento en que Habermas entró en el debate. No obstante, ante la posibilidad de futuros avances de las biotecnologías en la dirección de intervenciones en sujetos adultos, éste afirma que el criterio normativo al que tenemos que ceñirnos para evaluar su validez moral es siempre el mismo: el modo en que nos autocomprendemos como autores indivisos de nuestra biografía y en el que nos reconocemos unos a otros como seres con tal autonomía.

Así por ejemplo, podría pensarse que si las intervenciones pre-natales que mejoran las condiciones atléticas del recién nacido no son moralmente aceptables, según Habermas -más adelante veremos sus razones-, enton-

2 Así por ejemplo, Allen Buchanan resume el argumento habermasiano a "psicología de diván y palabrería retórica sobre la libertad» (Buchanan, 2011, pp. 5-6). 
ces tampoco lo deben ser las intervenciones somáticas que mejoren esas mismas condiciones en un adulto, si éstas pueden ser heredadas por su progenie. Por lo tanto, el argumento habermasiano cubriría directamente aquellos casos en que son afectadas generaciones que no toman por sí mismas la decisión de ser mejoradas, independientemente de que hayan sido realizadas en adultos responsables o en embriones. ¿Podría también Habermas dar respuesta a los problemas planteados por intervenciones no heredables practicadas sobre adultos como, por ejemplo, las prótesis cerebrales o físicas? ¿No afectan todas ellas, al fin y al cabo, a nuestra autocomprensión como sujetos modernos 3 ?

\subsubsection{La cuestión de la autonomía}

Antes de enfrentarse directamente al argumento habermasiano, parece necesario aclarar a qué se refiere éste con el término autonomía. En un primer momento, tanto la apelación que Habermas realiza a Søren Kierkegaard como su insistencia en que el sujeto modificado nunca se sentirá como autor indiviso de su biografía, parecen leer este concepto en clave de autenticidad -es decir, a la luz de la posibilidad que cada cual debe tener de escoger los modos de vida que, en términos del enfoque de las capacidades, cada uno tiene razones para valorar.

En una filosofía de raigambre kantiana como la de Habermas, la autonomía no puede quedar reducida a una cuestión como ésta, sino que tiene que ir más allá y hacer referencia a esa «facultad de la razón pura de ser por sí mismo práctica, (...) sometiendo la máxima de cada acción a las condiciones de aptitud para convertirse en ley universal» (Kant, 1989: 17). Aunque Habermas no define de modo explícito qué es eso que él entiende por autonomía en este texto de El futuro de la naturaleza bumana. No obs-

3 De hecho, el artículo de Peter Herissone-Kelly, citado con anterioridad, afirma que el argumento habermasiano, interpretado en función de lo que Michael Sandel propone en su texto Contra la perfección, casa mejor con el problema de las mejoras decididas por individuos adultos, que aquellas en las que éstos eligen por unos terceros. A su juicio, el adulto es quien por sí mismo trunca su autonomía, eligiendo recibir unas capacidades que no le son propias por nacimiento, sino introducidas artificialmente. Sin embargo, afirma este autor, en el caso del recién nacido, aunque es otra persona quien ha decidido cuáles serán sus capacidades, resulta que sí son aquellas con las que ha nacido, de modo que reflexivamente puede apoderarse de ellas y hacerlas propias, es decir, declararse responsable absoluto de ellas. Por el contrario, Matti Häyry defiende que J. Habermas sólo aceptaría las terapias relacionadas con intervenciones genéticas somáticas realizadas en adultos responsables. (Herrisone-Kelly, 2012; Häyry, 2012, p.214). 
tante, las referencias al ámbito moral y a la capacidad de regirnos por leyes universalizables son constantes (Habermas, 2002: 91).

Un texto en el que Habermas sí aclara perfectamente los diversos planos en que puede entenderse -y confundirse- el concepto de autonomía es «del uso pragmático, ético y moral de la razón práctica» (Habermas: 2004). Allí deja claro que "[a]utónoma sólo puede llamarse a la voluntad dirigida a razones morales y, por tanto, enteramente racional» (Habermas, 2004: 267). Pero esa capacidad de actuar de la razón práctica que denominamos autonomía puede entenderse en función de tres diversas estructuras del discurso: la pragmática, la ética, y la moral. En el primer caso, se hablaría de libertad de arbitrio de un sujeto, que toma decisiones inteligentes sobre la base de actitudes y preferencias de las que el sujeto parte contingentemente» (Habermas, 2004: 266). En el segundo, lo haríamos de capacidad de autorrealización del sujeto que está dirigido «a la capacidad de decisión existencial o de autoelección radical [que] opera siempre dentro del horizonte de la biografía» (Habermas, 2004: 266). Sólo en el caso del ámbito moral la voluntad es enteramente libre, es decir, autónoma, pues «se deja determinar por entero por leyes morales» (Habermas, 2004: 266). Así pues, hemos de distinguir entre libertad de arbitrio, autorrealización y autonomía.

La naturaleza dialógica de la ética habermasiana, frente a la monológica de la filosofía kantiana, obliga a entender ese «ser capaz de determinarse por leyes morales» desde la capacidad de formar parte y tomar partida en los discursos prácticos que especifican la naturaleza de lo justo, es decir, poseer la capacidad de ser interlocutores válidos. Esto sólo puede hacerse a través de instituciones justas que empoderen a los sujetos generando cada vez mayores espacios para la autonomía. Por ello, la autonomía no es congénita y natural en los seres humanos sino que se trata de algo que ha de ir generándose a través del tejido social, con el fin de proteger al individuo de su, esta vez sí, innata vulnerabilidad.

Según Habermas, lo moralmente relevante de las biotecnologías es que nuestra modernidad está en juego porque con su aplicación se ponen en peligro tres factores esenciales para nuestra autocomprensión como seres autónomos: a) no hacer propia nuestra biografía, sino haber sido moldeados; b) borrar la distinción entre cosas y personas, pues estas últimas se vuelven disponibles técnicamente al igual que las cosas; c) se establece una relación asimétrica entre las diversas generaciones, impidiendo que unas reconozcan a otras como seres de igual valor. Si alguna de estas tres 
condiciones no se da, entonces el liberalismo político pierde su razón de ser. Veamos cómo las recorre una a una el argumento habermasiano.

\subsubsection{Seres diseñados, no responsables de sus biografías}

El texto de Habermas comienza, en el prefacio, con una clara referencia a los prejuicios arraigados en nuestra conciencia moderna en relación a la «cría de humanos» (Habermas, 2002: 10). Una expresión que recuerda, por ejemplo, a ese primer capítulo de Un mundo feliz, en que Aldous Huxley narra todo el proceso por el que los seres humanos son diseñados a medida, con el fin de hacerlos pertenecer a una clase u otra, es decir, para poseer un determinado plan de vida y careciendo de la autonomía y responsabilidad de construirse su propia biografía. Ahí se concentran las ideas y temores en que se sustenta el texto habermasiano.

Sin embargo, aclara Habermas, que su argumento no es debido al simple hecho de poseer una dotación biológica diferente, sino «a la depreciación inducida de sí misma, al menoscabo de su autocomprensión moral» (Habermas, 2002: 106). No se trata de que por intervenir en la configuración biológica de los sujetos éstos dejen inmediatamente de ser autónomos, sino que una vez éstos se hacen conscientes del hecho de haber sido diseñados, nunca más podrán concebirse como seres responsables de sus biografías, pues han sido moldeadas por un tercero, que ha introducido sus intereses en su propia configuración biológica.

Así, no es éste un argumento sobre lo físico, sino que está basado en las intenciones y expectativas que los padres depositan en los hijos creados, y que éstos no podrán revisar críticamente por estar grabados a fuego en su código genético. Al nacer, afirma Habermas, el individuo comienza a ser artífice exclusivo de una vida que ha de apropiarse críticamente. Cuando existe un programador previo, éste se convierte en coautor de la biografía, de modo y manera que el nacimiento no es un comenzar desde cero, sino aquel lugar en que el programador te ha colocado. Sin embargo, este proceso no es algo automático, pues Habermas aclara que el problema no viene del mero hecho de intervenir, sino del influir en los planes vitales de una persona y que ésta sea consciente de ello.A su juicio, si bien en el caso de, por ejemplo, la educación que los padres ofrecen al niño, éste sí posee alguna posibilidad de negociar, responder, o librarse de ella, sin embargo, esta capacidad autónoma de respuesta es nula en lo que a la programación genética se refiere. 
Esta distinción entre el origen material y psíquico de la carencia de autonomía es la que se malinterpreta en el mundo anglo-americano, en el que suele reducirse el argumento habermasiano a una cuestión determinista. Un ejemplo de esta simplificación es el modo en que Allen Buchanan interpreta a Habermas:

Si el presupuesto de Habermas es que diseñar el genotipo en un embrión determina todas las características del individuo que se desarrolla a raíz de tal embrión, entonces él ha pasado por alto completamente la influencia del entorno. (Buchanan, 2011:5)

Buchanan contempla otras dos interpretaciones distintas del argumento habermasiano: una que tacha de "psicología de sillón pasada de moda» y otra que considera como un «error filosófico» (Buchanan, 2011: 5). La primera de éstas afirmaría que el argumento habermasiano se basa en el hecho psicológico de que el individuo, influido por el determinismo genético dominante en nuestra sociedad, no podría nunca considerarse como libre si supiera que ha sido creado. En el caso de la segunda interpretación, Buchanan entiende que Habermas afirma que el sujeto no podría configurarse a sí mismo de forma correcta como libre, incluso aunque en realidad cumpliera todas las condiciones para serlo y quisiera hacerlo, es decir, de hecho fuera libre.

Así pues, la crítica del norteamericano a Habermas está, principalmente, cimentada en determinismos que, estando ocultos en su argumentación, la hacen incorrecta, bien sean éstos de una naturaleza biológico-genética, psicológica -al más puro estilo del «pasado de moda» psicoanálisis- o filosófica. Sin embargo, el argumento, como ya hemos remarcado y seguiré haciendo a lo largo de este texto, puede entenderse más allá de cualquier forma de determinismo y comprenderse como una cuestión relativa a la autorrealización y el autorreconocimiento.

\subsubsection{La disposición técnica de lo basta abora inalcanzable. De personas $y$ cosas}

Afirma Victoria Camps que Habermas ha cometido el error de cimentar su argumento en una ontología pues, a su juicio, no parece que «sea preciso echar mano de una ontología de la persona, aunque sea una ontología moral» (Camps, 2002: 68). Por ello, le invita a limitarse a hacer uso del imperativo kantiano de la dignidad, como modo de mostrar a las personas dónde están los límites de su actuación libre en función de las consecuen- 
cias de la aplicación de las biotecnologías. Esta objeción planteada por la filósofa española debe enmarcarse dentro del segundo fundamento sobre el que se sustenta el argumento habermasiano: el de la disolución de las fronteras existentes entre las cosas y las personas.

Durante una parte de su argumentación, el filósofo alemán toma la distinción entre lo orgánico y lo inorgánico para afirmar que las biotecnologías presentan una amenaza para nuestra autocomprensión moral. Esta distinción clásica es aplicada a la comprensión del concepto de «cuerpo» elaborada en los estudios fenomenológicos de Helmuth Plessner, que distinguen entre ser cuerpo y tener cuerpo. Tenemos un cuerpo que habitamos, el cual vivimos como algo contingente y dado por naturaleza, pero, a su vez, somos un cuerpo con el que nos identificamos y al que vamos dándole forma, es decir, que se hace.

Con la aplicación de las biotecnologías de mejora humana esta distinción se viene abajo, pues nuestro cuerpo, que es lo crecido orgánicamente, se funde con lo hecho técnicamente, de forma que ambos no pueden distinguirse de un modo neto. A juicio de Habermas, cuando los individuos dejan de concebirse como algo con origen orgánico, no pueden desarrollar una existencia corporal válida. Habermas da un giro kantiano de este segundo punto, cuando afirma que con la intervención técnica de la integridad corporal de las personas se trata a éstas de un modo que no les es propio: como objetos o, más bien, como medios para ciertos fines -por ejemplo, las expectativas que el padre deposita en el hijo-, en vez de como seres valiosos en sí mismos que poseen dignidad -a diferencia de los objetos que tienen valor de uso. Con la aparición de los términos y fórmulas kantianas, Habermas prepara el camino para analizar el problema que le preocupa a través de la formulación que la ética discursiva hace del imperativo categórico.

Éste afirma que «únicamente se acepten como válidas aquellas normas que expresan la voluntad general, es decir, aquellas que han de poder convertirse en ley general» (Habermas, 1985: 83). Sin embargo, esta universalización de las normas no puede lograrse a través de un proceso monológico como el kantiano, que tiene su origen en la conciencia individual de cada uno, sino que debe ser elaborada de forma intersubjetivo-discursiva. El modo en que esto se realiza lo muestra el principio discursivo de su ética: "[ú]nicamente pueden aspirar a la validez aquellas normas que consiguen -o pueden conseguir- la aprobación de todos los participantes en cuanto participantes de un discurso práctico» (Habermas, 1985: 117). 
De este modo, una norma o acción sólo será correcta cuando pueda contar con el consenso de todos los afectados por las consecuencias de su aplicación. Este es el modo de solventar el problema de la instrumentalización de los individuos al que conduce, según Habermas, la aplicación de las nuevas técnicas de mejora. Los futuros hijos son, así, concebidos como segundas personas en un diálogo sobre las posibles consecuencias de dichas intervenciones, cuyas razones han de ser tenidas en cuenta. En palabras de Eduardo Mendieta, «sólo si somos capaces de imaginar argumentos con los que nuestras generaciones futuras podrían hipotéticamente consentir en que modificáramos su genotipo sería aceptable que lo hiciéramos» (Mendieta, 2002: 96).

Las generaciones futuras son interlocutores virtuales en el discurso moral -esta es una de las ventajas de las morales posconvencionales; las relaciones morales se extienden no sólo de forma horizontal, sino también vertical. De este modo, todos los posibles interlocutores válidos no pueden ser sometidos a relaciones coercitivas o estratégicas, sino que se debe presuponer su consentimiento virtual en cualquier modificación física que se les practique. A juicio de Habermas, sólo las intervenciones terapéuticas contarán con tal consenso anticipado, mientras que todo lo que vaya más allá de la lógica de la curación será problemático (Habermas, 2002: 73).

Acogiéndose a este argumento, Habermas rechaza el proyecto de aquellos defensores de la técnicas de mejora, como Allen Buchanan o Mark Coeckelbergh, que hablan de ciertas capacidades básicas humanas cuya mejora artificial siempre sería positiva ${ }^{4}$, pues así no sólo no se limitaría el horizonte de futuros proyectos de vida sino que, todo lo contrario, se capacitaría al sujeto para desarrollar otros proyectos de vida, que le habrían estado vedados de no haber sido mejorado técnicamente.

\subsubsection{Generaciones, castas y diferencia: relaciones asimétricas $y$ reconocimiento}

Si Un mundo feliz muestra lo problemático del no poderse concebir como autor indiviso y responsable de la vida propia, el Frankenstein de Mary Shelley apunta en la dirección del problema del no sentirse recono-

4 Así por ejemplo,A. Buchanan habla de «medios de interés general», mientras que M. Coeckelbergh trata de aplicar la teoría de las capacidades de Martha Nussbaum y Amartya Sen al problema (Buchanan et al, 2000: 167). 
cido por el hecho de ser considerado diferente, tanto por los otros como por uno mismo. En este sentido la filósofa Adela Cortina ha acertado al realizar una interpretación de la famosa obra de la novelista inglesa en clave del reconocimiento (Cortina, 2010).

Como afirma Cortina, el problema de la mejora es que podemos llegar a generar individuos con unas capacidades tales que, por su diferencia con los individuos no mejorados, no pueden autorreconocerse como iguales. Por lo tanto, de lo que se trata aquí, ya no es sólo de que no nos contemplamos como autores responsables y únicos de nuestra biografía, sino que, además, no podríamos ni siquiera concebirnos como personas de igual condición al resto, pues nuestra dignidad habría sido vulnerada. Otros autores como Allen Buchanan, Leon Kass y Nicholas Agar también han coincidido con Habermas en la centralidad del reconocimiento de la dignidad, como piedra de toque moral fundamental para analizar el caso de las tecnologías de mejora humana.

Siguiendo a Habermas, «la "dignidad humana” en estricto sentido moral y legal está ligada a [la] simetría de las relaciones [de forma que] sólo tiene algún significado en las relaciones interpersonales de reconocimiento recíproco» (Habermas, 2002: 50). Cuando la relación entre padre e hijo se vuelve asimétrica por culpa de la intervención técnica en la integridad corporal de este último, entonces el reconocimiento de su dignidad resulta imposible, pues la simetría ya se ha roto, el individuo programado no podrá sentirse como un igual, como digno, sino más bien como una cosa diseñada y creada. El problema de la mejora humana, que en Habermas comenzó como una cuestión de autonomía y experiencia corporal, se ha acabado convirtiendo en un problema relativo al reconocimiento de la dignidad de los individuos. De hecho, éste parece el modo más adecuado de enfrentarse a tal problema, es decir como algo propio del hombre moderno.

\section{EL ARGUMENTO HABERMASIANO MÁS ALLÁ DE LA ONTOLOGÍA Y DEL TERRENO POLÍTICO}

\subsection{Lo político y lo ontológico de la argumentación}

La filosofía de Habermas es posmetafísica, de ahí que a lo largo de su obra él se haya encargado de distinguir clara y tajantemente entre lo perteneciente al mundo de lo moral y al de lo ético. Si bien el primero está fundado en aquello que todos los individuos compartimos en tanto que 
seres humanos, a saber, nuestra capacidad discursiva, el segundo se refiere a las determinadas cosmovisiones en las que las comunidades morales se realizan, pues como bien afirma Habermas, más allá de las comunidades reales no existe nada, no hay un mundo nouménico al que pertenezcan las comunidades morales, sino que éstas se crean e insertan en formas de vida concretas, en los êthos de cada comunidad.

Este ámbito ético de la razón práctica, como lo denominará, por ejemplo, en su artículo ya citado sobre los usos de la razón práctica (Habermas, 2004), está relacionado con aquellos modos de vida privilegiados, cuya función es ofrecer una forma de autocomprensión existencial a los individuos. Por contra, lo moral se funda en aquello que todas y cada una de esas comunidades éticas guardan en común, es decir en los intereses de todos y cada uno en tanto que seres humanos. Así, por medio del desarrollo de una moral racional abstracta, es como él restringe el papel de la filosofía moral a la dilucidación de lo justo o lo correcto.

En este sentido, podemos afirmar que el argumento habermasiano es tanto ético como moral, pues si bien se basa en las condiciones de autocomprensión éticas de las sociedades modernas liberales actuales, resulta ser que éstas son las que incorporan el punto de vista moral en su êthos particular, pues conciben a sus miembros desde aquellas características comunes que todos y cada uno de ellos comparten por el mero hecho de ser seres humanos. Así, podemos afirmar con rotundidad que la interpretación política ofrecida por Eduardo Mendieta es errónea. Puede ser que éste tenga ramificaciones y consecuencias políticas -de hecho, no podría ser de otra manera cuando la normatividad de lo político se basa en la de lo moral. Sin embargo, que algo tenga consecuencias políticas no significa que sea de naturaleza exclusivamente política.

Cambiando de problema, nos encontramos con la posición defendida por Victoria Camps, quien había reclamado que un argumento de corte ontológico está fuera de lugar en la propuesta habermasiana e, incluso, en el problema ético referente a la mejora. Sin embargo, una antropología moral -cuya especificidad no cabe explicitar aquí- no parece algo descabellado a la hora de analizar un problema tan específico como éste, pues a la hora de entrar en contenidos tan especiales como los de la modificación de nuestra naturaleza necesitaremos también unos criterios morales, que yendo más a lo concreto sirvan como rasero moral de esa naturaleza humana que se quiere modificar. Esta es una metodología en la que ha insistido la llamada Escuela de Valencia, formada por los miembros del Departamento de Filosofía Moral de la Universidad de Valencia, y de la que, por supues- 
to, es un ejemplo Adela Cortina, que en su libro Las fronteras de la persona afirma:

[la] clave para el reconocimiento de la dignidad [es] el hecho de ser persona [en tanto que ésta es aquella que] tiene las capacidades requeridas para la autoconciencia, para el mutuo reconocimiento de la dignidad, para actuar desde la libertad y asumir su responsabilidad. (Cortina, 2009: 181, 185)

La dignidad va ligada al reconocimiento de la pertenencia a la especie humana, al hecho de ser persona, el cual nuestra filósofa caracteriza de la siguiente manera:

cualquier ser dotado de competencia comunicativa, en el sentido amplio de «comunicación» se nos descubre como un potencial participante en aquellos discursos cuyas decisiones le afectan. Y cualquier discurso práctico, para poder reclamar sentido y validez, presupone unos derechos de cuantos sean afectados por las decisiones que se toman en ellos. (Cortina, 2009: 218)

Estos derechos que Cortina reclama son los derechos pragmáticos de, primero, participar en discursos y, segundo, no ser obligado mediante coacción interna o externa.Además, éstos van ligados a otros derechos sin cuya protección sería imposible la realización de los pragmáticos: el derecho a la vida, las libertades básicas y las condiciones materiales y culturales, que permitan el desarrollo de los diálogos en igualdad de condiciones-derechos económicos, sociales y culturales.

De este modo, lo normativo que está a la base de nuestras sociedades democrático liberales puede ligarse a una antropología moral de los individuos. Como afirmaba Mendieta, lo normativo es un envoltorio que permite proteger y realizar esas capacidades humanas. Por ello, añade Cortina, «cualquier sociedad o cultura que dañe, instrumentalice o no potencie el desarrollo de los seres humanos [atendiendo a estas capacidades] está actuando de forma inmoral» (Cortina, 2009: 78). A través de una ética de la especie mínima como ésta se pueden establecer criterios normativos, que sirvan para guiarnos en un terreno tan complicado como el de la alteración de la naturaleza humana.

Otra cosa es que, como acertadamente señala Victoria Camps, el sentimiento individual de no ser un miembro digno de la especie sea suficiente como para que ello sea cierto y, por lo tanto, sea considerado seriamente desde un punto de vista normativo, a la hora de enfrentarse a la problemática que aquí se presenta (Camps, 2002: 66). Como afirma Axel Honneth, siguiendo la teoría hegeliana del reconocimiento: «la identidad del sujeto 
está $[\ldots]$ ligada al presupuesto de determinados actos de reconocimiento por parte de otros sujetos» (Honneth, 1997: 52). Así pues, el mero sentimiento por parte de un individuo de que carece de dignidad puede ser una condición necesaria para que dicha negación de la dignidad acontezca, pero no suficiente, pues bien puede ser que el individuo prefiera concebirse a sí mismo como víctima, cuando en realidad el resto no le está negando ningún tipo de autonomía o reconocimiento.

\subsection{Autonomía y asimetría: el argumento por continuidad}

El clásico argumento transhumanista en favor de las técnicas biomédicas de mejora humana es aquel que yo denomino como el argumento por continuidad. Según éste, lo novedoso ha de aceptarse si sus características morales relevantes coinciden con las de técnicas o intervenciones ya aceptadas. Pues, entonces, la única diferencia entre lo nuevo y lo aceptado sería, precisamente, que uno es novedoso y lo otro no, siendo el motivo del rechazo de las técnicas de mejora su novedad y no algún aspecto moralmente relevante diferente entre ambos.

Según estos autores, todas las técnicas y creaciones humanas comparten la cualidad moral de estar referidas al bienestar de los individuos porque tratan de hacer de sus vidas algo mejor. Si éste es también el fin de las técnicas de mejora humana, entonces ellas son moralmente idénticas a las tradicionales, por lo tanto, habremos de aceptarlas también. Este argumento ha sido denominado por Eric Parens como la «argumentación del ya lo hemos hecho antes y no ha sucedido nada» (Prusak, 2005: 35).

Este argumento por continuidad se aplica al caso de Habermas del siguiente modo. Las técnicas de mejora no parecen traer nada diferente a otros métodos que los padres usan para cuidar de sus hijos, como el entrenamiento o la educación. Es cierto que existen casos de híper-paternalismo, en los que los padres toman a niños que parecen muy bien dotados a la hora de realizar alguna actividad y restringen su vida a su cultivo, con ello la autonomía del futuro adulto se verá limitada por el plan de vida que sus padres han elegido. Al igual que esto puede suceder con el niño que es entrenado para ser un futbolista de élite o el que es educado para ser un músico virtuoso, también un padre puede elegir una dotación genética tan específica que vincule la existencia de su hijo a un plan de vida concreto. 
El mismo Habermas contesta a esta objeción presentada a través del argumento por continuidad afirmando que: «cuando [a dos prácticas] se les reprocha algo [...] por las misma razones, no puede recurrirse a una de las praxis para evitar a la otra los mismos reproches» (Habermas, 2002: 109). Es decir, la similitud entre unas prácticas y otras, y el hecho de que puedan conducir a unos mismos excesos, no debe, a su juicio, servir para igualarlas a todas desde un punto de vista moral. La continuidad puede ser engañosa, de modo que haría falta un examen único de las técnicas de mejora genética para mostrar tal continuidad y, a su vez, un examen de las razones morales por las que aceptamos las prácticas actuales, porque bien puede ser que éstas se acepten, simplemente, por hábito o costumbre, pero resulten ser inaceptables moralmente.

Nicholas Agar, como afirma Bernard G. Prusak, realiza tal análisis exclusivo de las técnicas biomédicas de mejora con el fin de mostrar dicha continuidad (Agar, 2004: 106). Para ello, apela a un caso real de programación genética para mostrar que, aún habiendo sido diseñados biológicamente los individuos, pueden hacer suya su propia biografía, es decir pueden hacer su biografía propia. Así, utiliza el ejemplo de Doron Blake. Éste fue concebido genéticamente en un laboratorio de selección genética usando los genes del donante $n^{\circ} 28$, que según su ficha era un profesor de ciencias que tocaba música clásica. El resultado fue positivo para Afton Blake, su madre, pues Doron era capaz de resolver problemas algebraicos y leer a Shakespeare durante su estancia en la guardería. La carrera de científico de éxito que ella quiso para él estaba casi asegurada, dadas estas condiciones de partida. Sin embargo, cuando Doron creció, rechazo ese plan de vida que su madre parecía haber seleccionado para él y dedicó su vida al estudio comparado de religiones.

Con un ejemplo así, Nicholas Agar pretende mostrar la continuidad entre las modificaciones externas y las internas, mostrando que estas últimas no tienen el peso moral tan grave que autores como Habermas quieren otorgarles y, por ello, son totalmente revisables desde el punto de vista de la autonomía del sujeto. Como afirma Adela Cortina (Cortina, 2002, p. 88), la asimetría de partida entre padre e hijo siempre se dará, tanto en procesos educativos como en intervenciones terapéuticas; lo que debemos de preocuparnos es de buscar criterios a los que recurrir, a la hora de evaluar si las decisiones de los padres han sido responsables con respecto a la autonomía y dignidad de sus hijos. Todos y cada uno somos hijos de nuestro tiempo y estamos obligados a heredar unas condiciones de las generaciones que nos han precedido, esa asimetría es inevitable (Mendieta, 2002: 
106). Sólo cabe esperar, como han reclamado autores como Adela Cortina, Victoria Camps, Jürgen Habermas, Karl Otto Apel o Hans Jonas, que las diversas generaciones actúen responsablemente teniendo en cuenta los intereses de sus sucesores.

\subsection{Determinismo genético}

Mostrada esta continuidad y similitud entre lo ya aceptado, las intervenciones externas, y lo novedoso, las intervenciones biomédicas de mejora, el discurso de Jürgen Habermas, a juicio de los transhumanistas, se convierte en una construcción alarmista, realizada además, como advierte Buchanan (2011), a través de una retórica oscura y complicada en torno a la autonomía, la igualdad y la identidad común de la especie. De hecho, la diferencia moralmente relevante entre modificaciones externas e internas, a juicio de estos autores, sólo parece sostenerse si se hace desde ese mismo determinismo genético que Habermas niega defender, pues sólo de esa manera el argumento por continuidad transhumanista dejaría de tener valor.

Así, una de las críticas más extendidas al argumento habermasiano es que éste presupone, para poder ser efectivo, el determinismo genético. El problema con este modo de determinismo es que no es ciencia, sino ideología. Así, autores como John Harris (2006: 138-142), Nicholas Agar (2004: 116-119) y Eduardo Mendieta han formulado sus críticas al alemán en esta dirección. Todo ello a pesar de que Habermas no se cansa de afirmar que quiere permanecer lejos del determinismo genético, y que su argumento nada tiene que ver con él.

En el análisis previo de su argumento pudimos comprobar que, aunque Habermas aseguraba que éste no tenía nada que ver con corrientes metafísicas concretas y referencia a límites naturales irrebasables, no obstante, no parecía cumplir con este requisito en su argumentación. Algo similar parece suceder respecto a su intento de no caer dentro del marco teórico del determinismo genético. Si bien afirma que los fundamentos de su argumento no son una cuestión genético-material, sino más bien algo referido a la resonancia psíquica de la modificación sobre el sujeto diseñado, paradójicamente, el determinismo parece muy presente.

Precisamente, esta problemática es a la que se ciñen los autores angloamericanos -reduciendo en muchas ocasiones el argumento habermasiano a ella- para criticarle que las personas mejoradas, por mucho que posean 
unas capacidades de partida distintas o superiores al resto, han de enfrentarse de igual modo a ese estar-arrojado-en-el-mundo que todos hemos de afrontar desde el momento del nacimiento. Esa experiencia primaria de ser-ahí-en-el-mundo- en la que la hermenéutica basó el sentimiento de individuación y responsabilidad con la vida propia y con la de aquellos otros con los que estamos irrevocablemente ligados- es idéntica, independientemente de la dotación genética de que cada uno disponga.

Como afirma Mendieta, parafraseando el lema de Gattaca, la famosa película de los años noventa, «el gen del destino no existe -y tampoco el de la libertad» (Mendieta, 2002: 105). Lo genético puede ser una base, tomando la distinción establecida por Adela Cortina (2011), que influencie en cierto modo nuestro comportamiento moral pero nunca será el fundamento del mismo -en términos leibnizianos, es condición necesaria pero no suficiente. Así, extraña que Habermas asegure con tanta firmeza que el sujeto dejaría de concebirse como autor indiviso de su vida, desde el mismo momento en que conozca que su biología de partida ha sido elegida en vez de recibida como fruto del azar de la lotería genética. Como afirma Cortina «[n]adie elige nacer, y ése no es un obstáculo a la autonomía» (Cortina, 2002).

Otra cosa muy distinta es que los otros no reconozcan como iguales a aquellos individuos que han sido genéticamente mejorados (Agar, 2004: 143-145). A mi juicio, ahí radica lo moralmente relevante y peligroso de esta cuestión. Sin embargo, por desgracia, Habermas parece desechar ese otro lado del reconocimiento referido al modo en que los otros conciben al sujeto programado -y, por supuesto, también en el que los sujetos diseñados conciben a los que no lo están.

\section{EN LA SENDA DEL ARGUMENTO HABERMASIANO}

A lo largo de este trabajo se ha logrado, primero, analizar y desmenuzar el argumento habermasiano en su totalidad (\$2) y, segundo, ponerlo a dialogar con sus críticos (\$3). Una vez cumplidos estos dos objetivos es cuando podemos ofrecer una evaluación global respecto al valor del argumento presentado por Jürgen Habermas en El futuro de la naturaleza bumana. Un texto que es ya un clásico, al que inevitablemente se tiene que acudir y con el que inexorablemente se tiene que dialogar, si se quiere entrar en el debate ético sobre la mejora humana. 
La concepción descalificativa del texto habermasiano como «oscuro, profundamente retórico y muy alemán» (Prusack, 2008: 32), que defienden autores transhumanistas como Allen Buchanan y John Harris, es falsa y parte, como ya afirmé, de una interpretación errónea y desinformada del mismo. Pues lo reduce a un problema de restricción de autonomía cuando, en realidad, Habermas está apuntando a algo más profundo, a una cuestión de identidad. De hecho, ése el mérito de su obra: ponernos en la pista de analizar el problema de la mejora como una cuestión de reconocimiento de nuestra identidad común.

En este sentido, acierta Peter Herissone-Kelly cuando defiende que el texto de Habermas sirve «como un mapa que muestra el área general en la que tenemos que mirar más que [para] ofrecer una lectura minuciosa y fiel de él» (Herrison-Kelly, 2012: 200-210). Debido al momento temprano del debate en que Habermas escribe su texto y, también, al miedo relacionado con la eugenesia que parece subyacer a su argumento, El futuro de la naturaleza bumana no es un texto que pueda aportar una comprensión completa y satisfactoria del problema de la mejora humana. Sin embargo, sí puede decirse que nos provee de intuiciones muy acertadas y prolíferas para el debate. En este sentido, puede decirse que el mentado carácter «muy alemán»o «muy de filosofía continental» del texto toma un sentido más que positivo, en vez de descalificativo.

A mi juicio, Habermas se quedó a medio camino, pues el problema central radica no sólo en el hecho de que el sujeto se sienta utilizado y, por tanto, irresponsable respecto a su biografía e incapaz de autorrealizarse, sino que esa percepción de sí mismo se corrobore con el modo en que los otros le conciben. $\mathrm{O}$, lo que es peor, que ésta venga infundida por el resto, que le conciban como un ser distinto y enfermizo. Valiéndome de las palabras de Axel Honneth, «no consiste solamente en la limitación violenta de la autonomía personal, sino en su conexión con el sentimiento de no poseer el estatus de un sujeto de interacción moralmente igual y plenamente valioso» (Honneth, 1997: 163). Por lo tanto, de lo que se trata es -siguiendo la terminología del principal representante de la tercera generación de la Escuela de Frankfurt- de que el individuo mejorado no sea objeto de menosprecio se le considere como un «muerto psíquico», un «muerto social» o un «enfermo» al que se le niega el reconocimiento.

El futuro de la naturaleza bumana de Habermas es un ponernos sobre la pista adecuada, por ello aún queda mucho camino por recorrer, si se quiere ofrecer un análisis moral esclarecedor del fenómeno de la mejora humana. Por ejemplo, habrá que ver en base a qué decimos que poseemos 
una dignidad o una identidad moral común y por qué merece nuestro reconocimiento. Así como también deberá mostrarse hasta qué punto el principio moral de la ética discursiva sirve por sí sólo para tratar completamente tal problema. Pues si, como el propio Habermas afirma, estamos ante una cuestión de ética de la especie, es decir de contenidos y análisis de la naturaleza humana, quizás otras corrientes filosófico-éticas que insistan en la necesidad de realizar una antropología del agente moral sean de mayor utilidad.

\section{BIBLIOGRAFÍA}

Agar, N. (2004): Liberal eugenics: In Defense of Human Enbancement, London, Blackwell Publishing.

Buchanan, A. (2011): Beyond Humanity? The ethics of biomedical enbancement, Oxford, Oxford University Press.

Buchanan, A.y otros (2000): From chance to choice: genetics and justice, Cambridge, Cambridge University Press.

CAMPs, V. (2002): «¿Qué hay de malo en la eugenesia?», Isegoria, 27, pp. 55-71.

Cortina, A. (2002): «Ética de las biotecnologías», Isegoría, 27, pp. 73-89.

- (2009): Las fronteras de la persona: el valor de los animales, la dignidad de los bumanos, Madrid, Taurus.

- (2010) «Frankenstein: el origen de la Neuroética», en El Pais, 17-102010.

- (2011): Neuroética y neuropolítica. Sugerencias para la educación moral, Madrid,Tecnos.

Elliot, C. (2003): Better than well:American Medicine Meets the American Dream, London, W.W. Norton.

Habermas, J. (1985): Conciencia moral y acción comunicativa, Barcelona, Península.[Trad. de Ramón García Cotarelo].

- (2002): El futuro de la naturaleza bumana: ¿Hacia una eugenesia liberal?, Barcelona, Paidós.

- (2004): Aclaraciones a la ética del discurso, trad. por. Manuel Jiménez Redondo, Argentina, El Cid Editor.

HARRIS, J. (2006): Enhancing evolution: the etbical case for making better people, Princenton (N.J.), Princenton University Press.

HäYry, M. (2012): «Protecting Humanity: Habermas and His Critics on the Ethics of Emerging Biotechnologies», Cambridge Quarterly of Healthcare Ethics, 21, pp. 211-222. 
Herissone-Kelly, P. (2012): «Habermas, Human Agency, and Human Genetic Enhancement», Cambridge Quarterly of Healthcare Ethics, 21, pp. 200-210.

HonNeTH, A. (1997): La lucha por el reconocimiento: por una gramática de los conflictos sociales, Barcelona, Crítica.

KANT, I. (1989): La metafísica de las costumbres, Madrid,Tecnos. [trad. por Adela Cortina y Jesús Conill].

Mendieta, E. (2002): «El debate sobre el futuro de la especie humana: Habermas critica la eugenesia liberal», Isegoría, 27, pp. 91-114.

Prusak, B. G. (2005): «Rethinking "Liberal Eugenics": Reflections and Questions on Habermas on Bioethics», The Hastings Center Report, 35, 6, pp. 31-42.

RIFKIN, J. (1999): The biotech century: bow genetic commerce will change the world, London, Penguin.

Turney, J. (1998): Frankenstein's footsteps: science, genetics and popular culture, New Haven, Yale University Press. 